\title{
A WEAKLY INFINITE-DIMENSIONAL SPACE WHOSE PRODUCT WITH THE IRRATIONALS IS STRONGLY INFINITE-DIMENSIONAL
}

\author{
ELŻBIETA POL
}

\begin{abstract}
We give an example of a weakly infinite-dimensional space $X$ such that the product $X \times B$ of $X$ and a subspace $B$ of the irrationals is strongly infinite-dimensional; under the assumption of the Continuum Hypothesis, $B$ can be the irrationals. This example answers a question of Addis and Gresham [AG].
\end{abstract}

1. Terminology and notation. All spaces under discussion are metrizable and separable. Our terminology follows [AP and E]. We denote by $I$ the real interval $[0,1]$, by $C$ the usual Cantor set in $I$ and by $I^{\omega}$ the Hilbert cube. We denote the space of the irrational numbers from $I$ by $P$ and the rational numbers from $I$ by $Q$.

A space $X$ is weakly infinite-dimensional [AP, Chapter 10, \$§4-7] if for every sequence $\left\{\left(A_{1}, B_{1}\right),\left(A_{2}, B_{2}\right), \ldots\right\}$ of pairs of closed disjoint subsets of $X$ there are partitions $L_{i}$ in $X$ between $A_{i}$ and $B_{i}$ such that $\bigcap_{i=1}^{\infty} L_{i}=\varnothing$. Otherwise, $X$ is strongly infinite-dimensional.

A space $X$ is called a $C$-space if for every sequence $\mathscr{G}_{1}, \mathscr{G}_{2}, \ldots$ of open covers of $X$ there exists a sequence $\mathscr{U}_{1}, \mathscr{U}_{2}, \ldots$ of families of open subsets of $X$ such that, for $i=1,2, \ldots$,

(i) the members of $\mathscr{U}_{i}$ are pairwise disjoint,

(ii) each member of $\mathscr{U}_{i}$ is contained in a member of $\mathscr{G}_{i}$,

(ii) the union $\bigcup_{i=1}^{\infty} \mathscr{U}_{i}$ covers $X$.

The notion of $C$-space was introduced by W. Haver in $[\mathbf{H}]$ for metric space and by D. Addis and J. Gresham in [AG] for general topological spaces.

LEMMA 1 [AG]. Every $C$-space is weakly infinite-dimensional.

2. Results. The aim of this note is to construct the following examples.

EXAMPLE 1. There exists a weakly infinite-dimensional space $X$ such that the product $X \times B$ of $X$ with a certain subspace $B$ of the space of irrationals is strongly infinite-dimensional. Moreover, $X$ is a $C$-space while $X \times B$ is not a $C$-space.

EXAMPLE 2. Under the assumption of the Continuum Hypothesis there exists a weakly infinite-dimensional space $X$ such that the product $X \times P$ of $X$ with the space of irrationals $P$ is strongly infinite-dimensional. Moreover, $X$ is a $C$-space while $X \times P$ is not a $C$-space.

Received by the editors June 18, 1985.

1980 Mathematics Subject Classification. Primary 54F45, 54B10.

Key words and phrases. Weakly infinite-dimensional spaces, products, property $C$. 
Example 2 gives, in particular, an answer to a question formulated by Addis and Gresham in $[\mathbf{A G}]$ as to whether the product of a $C$-space and the space of irrationals is a $C$-space.

The first example of two metrizable separable weakly infinite-dimensional spaces $X_{1}$ and $X_{2}$ (being also $C$-spaces) whose product $X_{1} \times X_{2}$ is strongly infinite-dimensional was given by R. Pol in [P2] (see [EP, Example 8.20] for the proof that $X_{i}$ are $C$-spaces).

3. Constructions. Our constructions are based on the following lemma, which follows an idea of Michael [M].

LemMA 2. Let $f: X \rightarrow Y$ be a mapping of a space $X$ into a space $Y$ and let $A=f^{-1}(B)$ for some $B \subset Y$. If the subset $\operatorname{Graph}(f \mid A)=\{(x, f(x)): x \in A\}$ of $X \times Y$ is strongly infinite-dimensional, then the product $X \times B$ is strongly infinite-dimensional (and thus is not a $C$-space).

The proof of this lemma follows from the fact that a closed subspace of a weakly infinite-dimensional space is weakly infinite-dimensional and that $X \times B$ contains $\operatorname{Graph}(f \mid A)$ as a closed subset.

Construction of Example 1. Let $T$ be the weakly infinite-dimensional compactum, which is not countable dimensional defined by R. Pol in [P1]. The space $T$ has the following structure: $T=Y \cup Z$, where $Y$ is a completely metrizable totally disconnected space, which is strongly infinite-dimensional, and $Z$ is the union of countably many 0-dimensional sets $Z_{1}, Z_{2}, \ldots$ Moreover, we can assume (see [P1, Comment B]) that $T$ is embedded in the product $C \times I^{\omega}$ of the Cantor set $C$ and the Hilbert cube $I^{\omega}$, in such a way that $Y$ is the graph of a certain first-Baire-class function $f$ : $C \rightarrow I^{\omega}$, i.e., $Y=\{(t, f(t)): t \in C\} \subset C \times I^{\omega}$.

Split the Cantor set $C$ into two disjoint Bernstein sets $B_{1}$ and $B_{2}$ (see $[K, \S 40]$ ), i.e., all compact subsets of $B_{i}$ are countable. Since $Y$ is strongly infinite-dimensional, either $Y_{1}=\left\{(t, f(t)): t \in B_{1}\right\}$ or $Y_{2}=\left\{(t, f(t)): t \in B_{2}\right\}$ is strongly infinite-dimensional. Suppose that $Y_{2}$ is strongly infinite-dimensional and let $X$ be the following subspace of $C \times C \times I^{\omega}: X=\left(B_{1} \times T\right) \cup\left\{(t, t, f(t)): t \in B_{2}\right\} \subset C \times T$. Denote $X_{1}=\left(B_{1} \times T\right), X_{2}=\left\{(t, t, f(t)): t \in B_{2}\right\}$. The set $X_{2}$ is obviously homeomorphic to $Y_{2}$, hence it is strongly infinite dimensional. We will show that

$X$ is a $C$-space.

Suppose that $\mathscr{G}_{1}, \mathscr{G}_{2}, \ldots$ is a sequence of open covers of $X$. Fix an $i \geqslant 3$. Since the set $B_{1} \times Z_{i-2}$ is 0 -dimensional, there exists a family $\mathscr{W}_{i}$ of pairwise disjoint sets open in $C \times T$ such that $B_{1} \times Z_{i-2} \subset \cup \mathscr{W}_{i}=W_{i}$ and for each $W \in \mathscr{W}_{i}$ the set $W \cap X$ is contained in some member of $\mathscr{G}_{i}$. Let $F=\left(B_{1} \times T\right) \backslash \cup_{i=3}^{\infty} W_{i}$; we will show that $\operatorname{dim} F=0$. Since $\bigcup_{i=3}^{\infty} W_{i} \supset B_{1} \times Z, F$ is a closed subset of $C \times T$ contained in $B_{1} \times Y$. Consider the projection $p_{1}: B_{1} \times T \rightarrow B_{1}$. Since $T$ is compact, $p_{1}$, as well as $p=p_{1} / F: F \rightarrow B_{1}$, is a closed mapping. Moreover, for each $t \in B_{1}$ the set $p^{-1}(t)=(\{t\} \times T) \cap F$ is a compact set contained in $\{t\} \times Y$. It follows that $\operatorname{dim} p^{-1}(t)=0$, because $Y$ is totally disconnected. Thus, by the Hure- 
wicz Theorem (see [E, Theorem 1.12.4])

$$
\operatorname{dim} F \leqslant \sup \left\{\operatorname{dim} p^{-1}(t): t \in B_{1}\right\}+\operatorname{dim} B_{1}=0 .
$$

Now, let $\mathscr{W}_{2}$ be a family of pairwise disjoint open subsets of $C \times T$ such that $F \subset \cup \mathscr{W}_{2}=W_{2}$ and for each $W \in \mathscr{W}_{2}$ there exists $U \in \mathscr{G}_{2}$ such that $W \cap X \subset U$. Put $K=X \backslash \bigcup_{i=2}^{\infty} W_{i}$; since $\bigcup_{i=2}^{\infty} W_{i} \supset B_{1} \times T$, then $K \subset X_{2}$. We shall show that the set $K$ is countable. Let $p_{2}: C \times T \rightarrow C$ be the projection. Since $p_{2}$ is a closed mapping, the set $L=p_{2}\left((C \times T) \backslash \bigcup_{i=2}^{\infty} W_{i}\right)$ is closed in $C$. Moreover, the set $L$ is contained in $B_{2}$, hence it is countable. It follows that the set $p_{2}(K) \subset L$ is countable, which implies that $K$ is countable, because $p_{2} \mid X_{2}: X_{2} \rightarrow B_{2}$ is a one-to-one mapping. Let $\mathscr{U}_{1}$ be a family of pairwise disjoint open subsets of $X$ such that $K \subset \cup \mathscr{U}_{1}$ and each member of $\mathscr{U}_{1}$ is contained in some member of $\mathscr{G}_{1}$. Then the family $\mathscr{U}_{1}, \mathscr{U}_{2}, \ldots$, where $\mathscr{U}_{i}=\left\{W \cap X: W \in \mathscr{W}_{i}\right\}$ for $i \geqslant 2$, satisfies conditions (i), (ii) and (iii).

Finally, let $f: X \rightarrow C$ be the projection. Then $f^{-1}\left(B_{2}\right)=X_{2}$ and the $\operatorname{Graph}\left(f \mid X_{2}\right)$ is homeomorphic to $X_{2}$ in a natural way, hence it is strongly infinite-dimensional. It follows by Lemma 2 that the product $X \times B_{2}$ is strongly infinite-dimensional. Since $B_{2}$ is a 0 -dimensional metrizable separable space, it is homeomorphic to a subset $B$ of the irrationals $P$.

Construction of Example 2. In this example we use, among others, some ideas of [P2].

Let $Z=\prod_{i=0}^{\infty} I_{i}$, where $I_{i}=I$ for $i=0,1,2, \ldots$, be the Hilbert cube and the mappings $p_{0}: Z \rightarrow I_{0}$ and $p_{n}: \prod_{i=1}^{\infty} I_{i} \rightarrow \prod_{i=1}^{n} I_{i}$ be appropriate projections. Arrange all rational numbers in $I_{0}$ into a sequence $q_{1}, q_{2}, \ldots$ Denote by $T$ a compactum obtained from $Z$ by attaching to each compactum $\left\{q_{n}\right\} \times \prod_{i=1}^{\infty} I_{i}$ the $n$-cube $\prod_{i=1}^{n} I_{i}$ by the map $p_{n}$, i.e., $T$ is a quotient space defined by an upper semicontinuous decomposition consisting of singletons $\{(t, x)\}$, where $t \in P$ and $x \in \prod_{i=1}^{\infty} I_{i}$, and the sets $\left\{q_{n}\right\} \times p_{n}^{-1}(y)$, where $y \in \prod_{i=1}^{n} I_{i}$. Let $\pi: Z \rightarrow T$ be the natural quotient mapping and $X_{1}=\pi\left(Q \times \prod_{i=1}^{\infty} I_{i}\right)$.

Using the Continuum Hypothesis, define a transfinite sequence $G_{1} \supset G_{2} \supset \ldots$ $\supset G_{\alpha} \supset \cdots, \alpha<\omega_{1}$, of $G_{\delta}$-subsets of $T$ containing $X_{1}$ and such that for every $G_{\delta}$-set $G$ in $T$ containing $X_{1}$ there exists $\alpha$ with $G_{\alpha} \subset G\left(\omega_{1}\right.$ denotes the first uncountable ordinal). Again by $\mathrm{CH}$, we can arrange all continua in $Z$ intersecting $\{0\} \times \prod_{i=1}^{\infty} I_{i}$ and $\{1\} \times \prod_{i=1}^{\infty} I_{i}$ into a sequence $K_{1}, K_{2}, \ldots, K_{\alpha}, \ldots, \alpha<\omega_{1}$.

By transfinite induction we will choose, for each $\alpha<\omega_{1}$, a point $y_{\alpha} \in K_{\alpha} \cap$ $\pi^{-1}\left(G_{\alpha}\right) \cap p_{0}^{-1}(P)$ such that $y_{\alpha} \notin\left\{y_{\beta}: \beta<\alpha\right\}$. Suppose that $\alpha=0$ or that we have already defined all points $y_{\beta}$ for $\beta<\alpha$. Since $\pi^{-1}\left(G_{\alpha}\right)$ is a $G_{\delta}$-set in $Z$ containing $Q \times \prod_{i=1}^{\infty} I_{i}, M=p_{0}\left(Z \backslash \pi^{-1}\left(G_{\alpha}\right)\right)$ is an $F_{\sigma}$-set in $I_{0}$, contained in $P$ (notice that the projection $p_{0}$ is closed). Thus the set $P \backslash M$ is of second category in $I_{0}$. Hence there exists $p_{\alpha} \in(P \backslash M) \backslash\left\{p_{0}\left(y_{\beta}\right): \beta<\alpha\right\}$. Since $\left\{p_{\alpha}\right\} \times \prod_{i=1}^{\infty} I_{i} \subset \pi^{-1}\left(G_{\alpha}\right)$ and the continuum $K_{\alpha}$ intersects $\left\{p_{\alpha}\right\} \times \prod_{i=1}^{\infty} I_{i}$, there exists $y_{\alpha} \in K_{\alpha} \cap \pi^{-1}\left(G_{\alpha}\right) \cap$ $p_{0}^{-1}\left(p_{\alpha}\right)$. If we put $x_{\alpha}=\pi\left(y_{\alpha}\right)$ for every $\alpha<\omega_{1}$, then

$$
x_{\alpha} \in G_{\alpha} \cap \pi\left(K_{\alpha}\right) \backslash\left(X_{1} \cup\left\{x_{\beta}: \beta<\alpha\right\}\right) .
$$


Claim. The subspace $X=X_{1} \cup\left\{x_{\alpha}: \alpha<\omega_{1}\right\}$ of $T$ has the desired properties. We will show that

$X$ is a $C$-space,

and

(3) the subspace $X_{2}=\left\{x_{\alpha}: \alpha<\omega_{1}\right\}$ of $X$ is strongly infinite dimensional.

To show (2) suppose that $\mathscr{G}_{1}, \mathscr{G}_{2}, \ldots$ is a sequence of open covers of $X$. Decompose the set $X_{1}$ into a sequence $Z_{1}, Z_{2}, \ldots$ of 0 -dimensional sets. For $i=2,3, \ldots$ choose a family $\mathscr{W}_{i}$ of disjoint open subsets of $T$ such that $Z_{i-1} \subset W_{i}$ $=\bigcup \mathscr{W}_{i}$ and for each $U \in \mathscr{W}_{i}$ there exists $G \in \mathscr{G}_{i}$ satisfying $U \cap X \subset G$ (see [E, Lemma 1.7.3]). Then $X_{1} \subset \bigcup_{i=2}^{\infty} W_{i}$ and there exists $\alpha$ such that $G_{\alpha} \subset \cup_{i=2}^{\infty} W_{i}$. The set $X \backslash \cup_{i=2}^{\infty} W_{i} \subset X \backslash G_{\alpha}$ is contained in $\left\{x_{\beta}: \beta<\alpha\right\}$, hence it is countable. Thus there exists a family $\mathscr{U}_{1}$ of open subsets of $X$ satisfying conditions (i) and (ii) for $i=1$ and such that $U_{1}=\bigcup \mathscr{U}_{1} \supset X \backslash \bigcup_{i=2}^{\infty} W_{i}$. The families $\mathscr{U}_{1}, \mathscr{U}_{2}, \ldots$, where $\mathscr{Q}_{i}=\left\{W \cap X: W \in \mathscr{W}_{i}\right\}$ for $i \geqslant 2$, satisfy conditions (i), (ii), and (iii).

To prove (3) we will show that the subspace $\pi^{-1}\left(X_{2}\right)$ of the Hilbert cube, which is homeomorphic to $X_{2}$, is strongly infinite dimensional. For $n=1,2, \ldots$, let $A_{n}=$ $\left\{\left\{x_{i}\right\} \in \prod_{i=0}^{\infty} I_{i}: x_{n}=0\right\}$ and $B_{n}=\left\{\left\{x_{i}\right\} \in \prod_{i=0}^{\infty} I_{i}: x_{n}=1\right\}$ be opposite faces of the Hilbert cube $Z$ and let $U_{i}$ and $V_{i}$ be open subsets of $Z$ such that $A_{i} \subset U_{i}$, $B_{i} \subset V_{i}$, and $\bar{U}_{i} \cap \bar{V}_{i}=\varnothing$. For $i=1,2, \ldots$, let $L_{i}$ be an arbitrary partition between $\bar{U}_{i} \cap \pi^{-}\left(X_{2}\right)$ and $\bar{V}_{i} \cap \pi^{-1}\left(X_{2}\right)$ in $\pi^{-1}\left(X_{2}\right)$. The partition $L_{i}$ can be extended (see [E, Lemma 1.2.9]) to a partition $L_{i}^{\prime}$ between $A_{i}$ and $B_{i}$ in $Z$ satisfying $L_{i}^{\prime} \cap \pi^{-1}\left(X_{2}\right)$ $\subset L_{i}$. Since $\bigcap_{i=1}^{\infty} L_{i}^{\prime}$ contains a continuum connecting $\{0\} \times \prod_{i=1}^{\infty} I_{i}$ and $\{1\} \times \prod_{i=1}^{\infty} I_{i}$ (see [RSW, Lemma 5.2]), say $K_{\alpha}$, we have $y_{\alpha} \in \bigcap_{i=1}^{\infty} L_{i}^{\prime} \cap \pi^{-1}\left(X_{2}\right)$. Therefore $\bigcap_{i=1}^{\infty} L_{i} \neq \varnothing$. This ends the proof of (3).

Finally, let $f: X \rightarrow I_{0}$ be the projection. Then $f^{-1}(P)=X_{2}$. Moreover, $\operatorname{Graph}\left(f \mid X_{2}\right)$ is homeomorphic to $X_{2}$, hence it is strongly infinite-dimensional. Thus, by Lemma 2 , the product $X \times P$ is strongly infinite-dimensional.

\section{REFERENCES}

[AG] D. F. Addis and J. H. Gresham, A class of infinite-dimensional spaces. Part I: Dimension theory and Alexandroff's problem, Fund. Math. 101 (1978), 195-205.

[AP] P. S. Alexandroff and B. A. Pasynkov, Introduction to dimension theory, Nauka, Moskva, 1973. (Russian)

[E] R. Engelking, Dimension theory, PWN, Warszawa, 1978.

[EP] R. Engelking and E. Pol, Countable-dimensional spaces: A survey, Dissertationes Math. 216 (1983), $1-41$.

[H] W. E. Haver, A covering property for metric spaces, Lecture Notes in Math., vol. 375, Springer-Verlag, Berlin and New York, 1974, pp. 108-113.

[K] K. Kuratowski, Topology, vols. I, II, PWN, Warszawa, 1966, 1968.

[M] E. Michael, The product of a normal space and a metric space need not be normal, Bull. Amer. Math. Soc. 69 (1963), 375-376.

[P1] R. Pol, A weakly infinite-dimensional compactum which is not countable-dimensional, Proc. Amer. Math. Soc. 82 (1981), 634-636.

[P2] _ A remark on A-weakly infinite-dimensional spaces, Topology Appl. 13 (1982), 97-101.

[RSW] L. R. Rubin, R. M. Shori, and J. J. Walsh, New dimension-theory techniques for constructing infinite-dimensional examples, General Topology Appl. 10 (1979), 93-103.

Department of Mathematics, University of Warsaw, Palac Kultury i Nauki, $00-901$ WARSZaWA, POLAND 